\title{
Validation of the Episodic Disability Framework with adults living with HIV
}

\author{
Kelly K. O'Brien, Steven Hanna, Sandra Gardner, \\ Ahmed M. Bayoumi, Sergio Rueda, Trevor A. Hart, \\ Curtis Cooper, Patricia Solomon, Sean B. Rourke, \\ Aileen M. Davis \& the OHTN Cohort Study Research Team
}

Version Post-Print/Accepted Manuscript

Citation O’Brien KK, Hanna S, Gardner S, Bayoumi AM, Rueda S, Hart T, (published version) Cooper C, Solomon P, Rourke S, Davis AM and the OHTN Cohort Study Team. Validation of the Episodic Disability Framework with adults living with HIV. Disability and Rehabilitation. 2014; 36(4): 319_ 329.

Publisher's Statement The Version of Record of this manuscript has been published and is available in Disability and Rehabilitation 2014. http://www.tandfonline.com/10.3109/09638288.2013.793408.

How to cite TSpace items

Always cite the published version, so the author(s) will receive recognition through services that track citation counts, e.g. Scopus. If you need to cite the page number of the TSpace version (original manuscript or accepted manuscript) because you cannot access the published version, then cite the TSpace version in addition to the published version using the permanent URI (handle) found on the record page. 


\section{Validation of the Episodic Disability Framework with Adults Living with HIV}

Kelly K. O’Brien ${ }^{1,2}$; Steven Hanna ${ }^{2,3}$; Sandra Gardner ${ }^{4}$; Ahmed M. Bayoumi ${ }^{5,6,7}$; Sergio Rueda ${ }^{4}$; Trevor A. Hart ${ }^{8,9}$; Curtis Cooper $^{10}$; Patricia Solomon ${ }^{2}$; Sean B. Rourke ${ }^{4,5,11}$; Aileen M. Davis ${ }^{6,12}$ and the OHTN Cohort Study Research Team*

1 Department of Physical Therapy, University of Toronto, Toronto, Ontario, Canada

2 School of Rehabilitation Science, McMaster University, Hamilton, Ontario, Canada

3 Department of Clinical Epidemiology and Biostatistics, McMaster University, Hamilton, Ontario, Canada

4 The Ontario HIV Treatment Network, Toronto, Ontario, Canada

5 Centre for Research on Inner City Health, St. Michael's Hospital, Toronto, Ontario, Canada

6 Institute of Health Policy, Management and Evaluation, University of Toronto, Toronto, Ontario, Canada

7 Department of Medicine, University of Toronto, Toronto, Ontario, Canada

8 Ryerson University, Department of Psychology, Toronto, Ontario, Canada

9 Dalla Lana School of Public Health, University of Toronto, Toronto, Ontario, Canada

${ }^{10}$ University of Ottawa, Division of Infectious Diseases, The Ottawa Hospital

Ottawa, Ontario, Canada

${ }^{11}$ Department of Psychiatry, University of Toronto, Toronto, Ontario, Canada

${ }^{12}$ University Health Network, Toronto Western Research Institute, Toronto, Ontario, Canada

*The OHTN Cohort Study Research Team consists of Dr. Sean B. Rourke (Principal Investigator, University of Toronto and OHTN), Dr. Ann Burchell (Co-Principal Investigator, 
OHTN), Dr. Sandra Gardner (OHTN), Dr. Sergio Rueda (OHTN), Dr. Ahmed Bayoumi and Dr. Kevin Gough, St. Michael's Hospital; Dr. Jeffrey Cohen, Windsor Regional Hospital; Dr. Curtis Cooper, Ottawa General Hospital; Dr. Don Kilby, University of Ottawa Health Services; Dr. Mona Loutfy and Dr. Fred Crouzat,, Maple Leaf Medical Clinic; Dr. Anita Rachlis and Dr. Nicole Mittmann, Sunnybrook Health Sciences Centre; Dr. Janet Raboud and Dr. Irving Salit, Toronto General Hospital; Dr. Edward Ralph, St. Joseph's Health Care; Dr. Roger Sandre, Sudbury Regional Hospital; Dr. Marek Smieja, Hamilton Health Sciences, McMaster University Medical Centre; and Dr. Wendy Wobeser, Hotel Dieu Hospital. 


\title{
Validation of the Episodic Disability Framework with Adults Living with HIV
}

\begin{abstract}
Purpose: To assess the validity of dimensions of disability in the Episodic Disability

Framework, a conceptual framework derived from the perspective of adults living with HIV.

Methods: We conducted confirmatory factor analyses with 913 adults living with HIV in an observational cohort study called the Ontario HIV Treatment Network Cohort Study (OCS). We tested hypotheses that dimensions of disability in the Episodic Disability Framework were represented by a group of measured variables in the observational database.
\end{abstract}

Results: A model comprised of four latent variables and 43 indicator variables with one crossloading was superior to models with fewer latent variables and more indicator variables and supported the validity of disability dimensions: physical health symptoms (represented by 21 indicator variables), mental health symptoms (10 variables), difficulties with day-to-day activities ( 5 variables), and challenges to social inclusion ( 8 variables). Overall goodness of fit statistics were $\chi^{2}=2621.50(p<0.001)$, Comparative Fit Index $=0.912$, Tucker Lewis Index $=0.907$ and Root Mean Square Error of Approximation=0.048. Dimensions of disability correlated with each other ranging from $\mathrm{r}=0.44$ (between physical symptoms and challenges to social inclusion) to $\mathrm{r}=0.81$ (between physical symptoms and difficulties with day-to-day activities).

Conclusions: This study supports the validity of four disability dimensions in the Episodic Disability Framework. This framework provides a new way to conceptualize disability and can lay the foundation for developing a future HIV disability measure for clinical and health services research. 


\section{INTRODUCTION}

With improved access to combination antiretroviral therapy, many people living with Human Immunodeficiency Virus (HIV) are living longer [1, 2]. However, individuals can face a variety of health-related consequences of HIV, comorbidities, and the potential side effects of treatment [3-7]. Health challenges experienced by adults living with HIV are complex and can range from symptoms such as fatigue, weakness, pain, and changes in body composition to difficulties participating in society, such as employment $[5,8-10]$. Collectively, these health-related challenges may lead to disability.

In an earlier phase of research, we used qualitative methods to develop the Episodic Disability Framework, a new conceptual framework of disability derived from the perspective of 38 adults living with HIV [11]. In this Framework, disability is multi-dimensional and episodic characterized by periods of wellness and illness. The Episodic Disability Framework consists of three main components. The first component includes dimensions of disability: a) symptoms and impairments (e.g. physical and mental health-related challenges), b) difficulties carrying out dayto-day activities (e.g. difficulties with walking, activities of daily living, carrying out household chores), c) challenges to social inclusion (e.g. challenges fulfilling roles as a parent, difficulties engaging in work and school, personal relationships), and d) uncertainty about future health (e.g. worrying about the future and the impact of uncertainty on life decisions) [11]. The second component includes contextual factors: extrinsic factors (social support and stigma) or intrinsic factors (living strategies and personal attributes) that can exacerbate or alleviate dimensions of disability [12]. The third component includes triggers, which are defined as life events that mark major or momentous episodes of disability. Examples include receiving notification of an HIV diagnosis, starting or changing antiretroviral medications, and experiencing a serious illness [11]. 
The Episodic Disability Framework goes beyond existing frameworks such as the Disablement Process or International Classification of Functioning, Disability and Health (ICF) which were developed prior to the advent of combination antiretroviral therapy or were not developed specifically from the perspectives of adults living with HIV [13-18]. Distinct features of the Framework include the description of disability as episodic in nature, the detailed inclusion of contextual factors that influence disability, and the consideration of life events that may initiate a major episode. The Episodic Disability Framework may be used by clinicians, adults living with HIV, representatives from community-based service organizations, and researchers to better understand and articulate episodic disability experienced by adults living with HIV. However, the validity of this framework has not been empirically tested.

In this study, we assessed the construct validity of dimensions of disability in the Episodic Disability Framework. Specifically, we determined whether dimensions of disability within the Framework were represented by a group of measured variables in a large observational cohort of people living with HIV. Validating the Episodic Disability Framework will establish a strong conceptual foundation from which to guide the comprehensive assessment of disability, indicate areas to apply rehabilitation interventions that may prevent or mitigate disability, and establish a future measure of disability experienced by adults living with HIV.

\section{METHODS}

We evaluated the validity of the Episodic Disability Framework using data from the Ontario HIV Treatment Network Cohort Study (OCS) $[19,20]$. The OCS is an ongoing longitudinal multi-site clinic-based observational cohort study that has collected clinical and socio-behavioural data from HIV-positive adults who are residents of Ontario and provided informed consent to understand the psychosocial, behavioural and health context of people living with HIV and their 
patterns of health services use [19]. Participants complete an interviewer-administered questionnaire at yearly intervals. We focused on participants in three sites in Toronto, Ontario (Toronto General Hospital, St. Michael's Hospital and Sunnybrook Health Sciences Centre) who completed a 90 to 120 minute questionnaire comprised of detailed health status instruments [2129] and questions related to employment status, housing, and income. This study was approved by the OCS Governance Committee and by Research Ethics Boards at McMaster University, Hamilton, Ontario, and Ryerson University, Toronto, Ontario, Canada.

\section{OCS Measures}

Variables in our measurement model included a combination of summary, subscale and item scores from the OCS questionnaire. The HIV Symptom Index is a 20 -item questionnaire that describes the presence and burdensome nature of symptoms experienced by adults with HIV. Each item is scored using a five point ordinal scale ranging from 'I do not have this symptom' to 'I have this symptom and it bothers me a lot'. The HIV Symptom Index demonstrates good construct validity with people living with HIV [26] and developers advise interpreting each symptom separately rather than calculating summary scores. The Center for Epidemiologic Studies for Depression Scale (CES-D) is a 20-item instrument that measures depressive symptomatology [23]. The Scale possesses high internal consistency, adequate test-retest reliability and concurrent and construct validity in the general population [23] and has been used extensively with people living with HIV [30-34]. Item scores can be added to generate a summary score, ranging from 0-60. Higher scores indicate more symptoms and higher frequencies of experiencing depressive symptoms [23]. The Health Utilities Index (HUI) is a generic utility index designed to measure health status and health-related quality of life [22, 35]. Scores range from zero to one with higher scores representing better health. The HUI is 
comprised of 41 items, from which domain scores may be calculated for vision, hearing, speech, dexterity, pain, emotion, cognition, mobility, self-care, and ambulation [22, 35]. The SF-36 questionnaire is a generic health-related quality of life instrument $[21,36]$. The measurement properties of the SF-36 are well established, demonstrating high internal consistency reliability, content, construct, and predictive validity [24, 25]. Domain scores for the SF-36 include physical function, role limitation physical, bodily pain, vitality, role limitation emotional, mental health, social function, and general health perception that range on a scale from $0-100$ with higher scores indicating better quality of life. The Ongoing Problems Stress Index is a component of the National Population Health Survey Stress Questionnaire. The index includes 17 statements that pertain to stressful situations asking whether the statement is true or false. Total scores can range from $0-17$ with higher scores representing increased stress [28, 37]. The Medical Outcomes Study Cognitive Functioning Scale includes four items with a six point ordinal scale that measures challenges across reasoning and problem solving, memory, attention, and concentration and thinking [29]. The MOS Cognitive Function Scale scores range from 0100 with higher scores representing better cognitive function. The scale has been validated for use with people living with HIV [38]. The EQ-5D EuroQoL Questionnaire is a standardized general measure of health related quality of life. The EQ-5D is comprised of five items (mobility, self-care, usual activities, pain and discomfort, and anxiety and depression), each of which is coded on a three point ordinal scale ranging from no problems to severe problems [27, 39]. The EQ-5D demonstrated good construct validity with people living with HIV [40].

Remaining indicator variables for our measurement model were individual items generated specifically for the OCS questionnaire including housing, employment, education, income and 
occupation. This questionnaire was developed and pre-tested with extensive consultation with HIV researchers and community members across Ontario.

\section{Analysis}

Our purpose was to assess the construct validity of dimensions of disability in the Episodic Disability Framework, a conceptual framework derived from the perspective of adults living with HIV. We conducted a confirmatory factor analysis to assess the construct validity of the dimensions of disability in the Episodic Disability Framework. Specifically, we used crosssectional data to test hypotheses that dimensions of disability (latent variables) in the Framework were represented by observed measures in the OCS (indicator variables). We allocated indicator variables to latent variables based on categories that comprised each dimension in the Episodic Disability Framework [11, 41]. We further divided symptoms and impairments into physical and mental health symptoms, because while correlated, these variables differ conceptually [42]. Uncertainty was not considered in our analysis because no measures in the OCS captured this dimension. See figure 1 for our a priori measurement model.

\section{Insert figure 1 about here}

We assessed convergent construct validity by determining the extent to which variables from the OCS represented a hypothesized dimension of disability with factor loadings $>0.30$. We assessed divergent construct validity by determining the extent to which dimensions of disability were distinct constructs that together comprised the larger construct of disability. We considered correlations between latent variables $<0.80$ as signifying distinct dimensions of disability [43]. 
Sample Size Estimation

At least five observations per unknown parameter estimate is required for confirmatory factor analysis $[43,44]$. Our primary hypothesized model had 120 unknown parameter estimates (not shown) that included factor loadings and error variances for each of the indicator variables $(n=110)$ plus latent variable variances and covariances $(n=10)$. Hence, we required at least 600 observations for our analysis.

\section{Confirmatory Factor Analysis}

We used Mplus version 6.0 statistical software and the weighted least squares method of estimation, which is appropriate for combinations of continuous and categorical variables and non-normally distributed data [45]. Prior to our analysis, we recoded all 'don't know' or 'refused' item responses to missing values. We assessed the mean, standard deviation, skewness, kurtosis, and frequency of missing values for each variable. All variables were recoded such that higher scores indicated greater disability. Where possible, we included subscale or summary scores to simplify the measurement model. We estimated and reported standardized parameter estimates (or factor loadings) given the variables possessed different units of measurement. Using the weighted least squares method of estimation, missing data were dealt with using pairwise deletion [45].

\section{Confirmatory Factor Models}

In our initial model, we included all variables that we hypothesized represented the dimensions of disability in the Episodic Disability Framework (figure 1). Confirmatory factor analysis involves a complex estimation of parameters that include factor loadings, variances, covariances and measurement error for indicator and latent variables [43]. Each of these parameters can be freely estimated (derived from the analysis) or fixed (assigned a value by the researcher) and 
indicator variables also can be allowed to cross load (represent multiple latent variables) [44]. For our analysis, all error variances were freely estimated. All error covariances were initially fixed to zero and variables were allowed to load on only one latent variable. Latent variables were allowed to correlate freely. We systematically modified the model to reduce complexity and improve fit where possible by removing variables that measured duplicate categories of disability (were highly correlated), lacked variability in scores (e.g. ceiling or floor effect), or when item removal made theoretical and clinical sense. We cross loaded variables, allowing them to load on more than one latent variable, if this made theoretical and clinical sense and the modification indices suggested a large improvement in fit [43].

We used a combination of approaches to evaluate the confirmatory factor analysis (CFA) solution including overall goodness of fit, localized areas of strain (areas of misfit in the solution), and interpretability (magnitude and statistical significance of the parameter estimates) [43]. If the CFA solution demonstrated adequate goodness of fit we considered this as construct validation of the disability dimensions in the Episodic Disability Framework [11]. Overall Goodness of Fit: We considered a Root Mean Square Error of Approximation (RMSEA) $<0.05$, Comparative Fit Index $(\mathrm{CFI}) \geq 0.95$, and Tucker Lewis Index (TLI) $\geq 0.95$ to indicate good model fit $[46,47]$. We considered the RMSEA as the primary statistic for overall goodness of model fit because it is recommended for confirmatory factor analytical contexts [48]. We reported the chi square statistic $\left(\chi^{2}\right)$ but did not consider it a determinant of model fit given its sensitivity to large sample sizes, which can overestimate lack of model fit [43].

Localized Area of Strain: We assessed areas of misfit in the solution by reviewing modification indices (values that indicate how much the chi square statistic would decrease if fixed parameters were allowed to be freely estimated) to identify ways to improve model fit. However, we only 
made modifications if they made theoretical and clinical sense [49].

Parameter Estimates: We considered standardized coefficients (i.e., factor loadings) $\geq 0.30$ $(\mathrm{p}<0.05)$ as 'representing' a given dimension of disability [43]. We used chi square difference tests where possible to identify statistically significant improvements in model fit.

We evaluated six CFA solutions. Our primary hypothesized model (Model 1) included a four factor model with all possible OCS variables. Model 2 similarly included all possible variables but had three factors as we collapsed the physical and mental health symptoms into one latent variable. Models 3 and 4 were simplified models with four and three latent variables respectively. We removed variables that lacked variance, were duplicate measures of disability (i.e., measured the same construct), or when their removal made clinical or theoretical sense. Models 5 and 6 included cross loading the SF-36 social function subscale score across three (Model 5) and two (Model 6) dimensions of disability.

\section{RESULTS}

We studied 913 adults living with HIV who completed OCS questionnaires between October 2007 and March 2009 in Toronto, Ontario. Participants were primarily male (83\%), taking combination antiretroviral therapy $(86 \%)$, with a median age of 47 years and median length of time since diagnosis of 11.2 years (table 1$)$.

\section{Insert table 1 about here}

\section{Indicator Variables \\ Indicator variables in our original hypothesized model included 37 item, 17 subscale, and three summary scores that spanned continuous $(n=24)$ and discrete ordered categorical $(n=33)$ levels of measurement (table 2). Skewness and kurtosis values (not shown) indicated non-normality of}


the data, supporting our use of the weighted least squares method of estimation.

\section{Insert table 2 about here}

\section{Confirmatory Factor Analysis Solutions}

Results of all CFA solutions are presented in table 3. Correlation matrices are available on request. Of all the models tested, the four latent variable model with 43 indicator variables and cross loading of the SF-36 social function subscale score onto the physical and mental health symptom and impairment dimensions provided the best fit to the data (Model 6).

Our initial model, Model 1, consisted of four latent variables including physical symptoms and impairments (represented by 25 indicator variables), mental health symptoms and impairments (11 indicator variables), difficulties with day-to-day activities (8 indicator variables), and challenges to social inclusion (13 indicator variables) (table 2). Model 2 consisted of three latent variables represented by 57 indicator variables whereby physical and mental health symptoms and impairments were collapsed into one latent variable. The difference between Models 1 and 2 was significant [chi square difference test $(\mathrm{df})=302.14(3), \mathrm{p}<0.001$ ], indicating that Model 1 with four latent variables possessed better fit than Model 2 (table 3).

Model 3 was a simplified model with 43 indicator variables across four latent variables. Fourteen variables were removed from Model 1 because they were highly skewed, lacked variance, were highly or lowly correlated with other variables in the model, duplicated other measures in the model, or because removing the variable improved model fit as well as made clinical and theoretical sense in accordance with the Episodic Disability Framework. We were unable to use a likelihood ratio (i.e., chi-square difference) test to compare Model 3 with Model 1 because the former was not nested within the latter. However, overall goodness of fit statistics for Model 3 improved across all indices including a reduction in the chi square statistic, $\left[\chi^{2}(\mathrm{df})\right.$ : 
3077.75 (854)], increase in CFA (0.889) and TLI (0.883) and reduction in RMSEA (0.053) (table

3). These values still did not achieve our criteria for goodness of fit. High modification indices ( $>250)$ suggested the chi square statistic could decrease by more than 250 if the SF-36 social function summary score were free to load on both physical and mental health symptoms and impairments. Chi square difference testing of Model 4 versus Model 3 further confirmed that a four latent variable model better represented the data (table 3).

Model 5 included cross-loading of the SF-36 social function subscale score on physical and mental health symptoms and impairments and challenges to social inclusion. In addition to the modification indices (which indicated the overall chi square statistic would decrease by more than 250 if the SF-36 social function summary score were free to load on both physical and mental health symptoms and impairments), cross loading was considered reasonable based on the phrasing of the subscale items that asked: 'to what extent has your physical and emotional problems interfered with your social activities?' and 'how much time has your physical and emotional problems interfered with your social activities?' We first ran a null model with cross loading the SF-36 social function subscale score across three latent variables (physical symptoms and impairments, mental health symptoms and impairments, and challenges to social inclusion) (Model 5). Results (not shown) showed the SF-36 social function subscale significantly loaded on the symptoms and impairments dimensions only and did not load on challenges to social inclusion. Chi square difference testing that compared the Model 5 (cross loading on three dimensions) to Model 6 (cross loading on the two symptoms and impairments dimensions only) was not significant $\left[\chi^{2}(\mathrm{df}): 0.258(1) ; \mathrm{p}=0.612\right]$ indicating that the nested model (Model 6) did not significantly worsen the fit (table 3 ). Hence, overall goodness of fit in combination with interpretability of the parameter estimates indicated that Model 6 was the best and final model to 
represent dimensions of disability in the Episodic Disability Framework.

Insert table 3 about here

\section{Final Model - Dimensions of Disability}

The final model was comprised of four dimensions of disability and 43 indicator variables (Model 6) (table 4). Overall goodness of fit statistics were $\chi^{2}=2621.50(p<0.001)$; CFI $=0.912$, $\mathrm{TLI}=0.907$, and RMSEA $=0.048$, achieving our a priori criterion for goodness of fit (RMSEA). All the indicator variables significantly loaded on their hypothesized dimensions (factor loadings $\geq 0.30$ ). To interpret the first factor loading in table 4 , one standard deviation increase in physical symptoms and impairments is associated with a 0.78 standard deviation increase in loss of energy. Alternatively, the correlation between loss of energy and physical symptoms and impairments is 0.78 .

\section{Insert table 4 about here}

The SF-36 social function subscale score cross-loaded on the physical symptoms $(0.21)$ and the mental health symptoms dimensions (0.61). The latent variables (dimensions of disability) were correlated with each other, ranging from $r=0.44$ (between physical symptoms and impairments and challenges to social inclusion) to $r=0.81$ (between physical symptoms and impairments and difficulties with day-to-day activities) (figure 2).

\section{Insert figure 2 about here}

\section{DISCUSSION}

This is the first study to empirically assess the validity of the Episodic Disability Framework, a conceptual framework of disability developed from the perspective of adults living with HIV. Results of our confirmatory factor analysis that demonstrated good overall model fit support the validity of the dimensions of disability in the Episodic Disability Framework [11]. Although 
evidence exists supporting the validation of quality of life frameworks with HIV populations [51, 52], this is the first known validation of an HIV-specific framework of disability.

Our initial model included all possible variables that represented disability in the OCS questionnaire. This initial approach led to an overwhelmingly complex model comprised of duplicate measures of disability. By including summary and subscale scores where possible and removing variables where the process made statistical and clinical sense, we were able to simplify the model. In the final model, dimensions of disability were represented by 35 indicator variables derived from six HIV-specific and generic health status measures as well as eight indicator variables derived from single items (table 4). Existing validated health status measures represented the symptoms and impairments and difficulties with day-to-day activities dimensions, whereas the challenge to social inclusion dimension was represented by individual items in the OCS questionnaire. These observations support results from a content analysis that suggested challenges to social inclusion experienced by adults living with HIV were less well represented in existing health status instruments compared with symptoms and impairments [41].

All indicator variables significantly loaded on their hypothesized dimensions of disability (factor loadings $\geq 0.30$ ). We allowed the SF-36 social function subscale scores to load on both physical symptoms (0.21) and mental health symptoms dimensions $(0.61)$. Despite the factor loading on physical symptoms was $<0.30$, we retained this cross loading in the model for two reasons. First, allowing the SF-36 social function summary score to load on both physical and mental health symptoms and impairments improved the model fit by reducing the RMSEA from 0.053 to 0.048 (not shown). Second, we considered this cross loading to be reasonable because the subscale items included phrasing that referred to how physical and emotional problems interfered with social activities. 
Correlations between the latent variables ranged from $r=0.44$ to $r=0.81$. High correlations existed between physical symptoms and difficulties with day-to-day activities (r $=0.81)$ and physical and mental health symptoms $(\mathrm{r}=0.79)$ suggesting that these latent variables may not be empirically distinct [43]. However, these dimensions of disability were represented by variables that contained items with similar ordered categorical response options such as the HIV Symptom Index and EQ-5D questionnaire, which could explain the high correlations (0.790.81) between these dimensions and obscure the discriminant nature between physical and mental health symptoms and impairments and difficulties with day-to-day activities. Hence, we concluded there were four dimensions that comprise the larger construct of disability. The mental health symptoms dimension combined mood (SF-36 mental health subscale score, CES-D) and cognition (MOS cognitive function score) variables. Mood states included items related to depression, and cognition included items related to difficulty focusing attention, problem solving and memory problems. These components of mental health, when measured subjectively, correlate with each other, supporting our decision to collectively consider mental health symptoms as one latent variable in the model $[42,53]$. However, treatment strategies to address mental health symptoms may differ depending on whether the impairments are mood or cognition related, suggesting these may be distinct clinical concepts. Future research involving an exploratory factor analysis may help to determine whether mood and cognition should be considered collective or distinct domains (dimensions) in a future measure of disability.

OCS variables possessed different characteristics of measurement which could influence item responses, factor loadings and overall model fit. For example, the timeframe of OCS measures varied, asking about health states within the past four weeks (HIV Symptom Index, SF36 questionnaire, MOS-HIV Cognitive Function Scale), within the past week (CES-D), or about 
today (EQ-5D). Given health states among people living with HIV may fluctuate on a weekly or even daily basis, responses to item measures may differ depending on the timeframe posed in the question. High correlations between items may occur because of similar timeframes or response options rather than their content as it relates to disability. However, we chose to retain these measures in the model, specifically the EQ-5D subscales in order to fulfill the three indicator variable minimum required for each latent variable in CFA, which was required for the difficulties with day-to-day activities dimension [43].

Decisions for using item, subscale, or summary scores in the model could influence factor loadings and overall indices of model fit. We based our decisions on the construct measured as it related to the Episodic Disability Framework as well as scoring recommendations established by authors of the original instruments. For example, we used specific items in the HIV Symptom Index to represent dimensions in the Framework because this instrument does not possess formal summary (or subscale) scores [26]. We used subscale scores of the EQ-5D and SF-36 questionnaires because they were developed to measure multi-dimensional constructs of health status or health-related quality of life $[21,22,27]$ and contain domains that we hypothesized represented different dimensions of disability. We used summary scores from the CES-D, Medical Outcomes Study-HIV Cognitive Functioning Scale and Ongoing Problems Stress Index because collectively they measured constructs of depressive symptomology [23], cognition [50] and stress[28] respectively, all of which we hypothesized represented the mental health symptoms dimension of disability.

When validating the Episodic Disability Framework, it is important to consider the context in which the framework was derived and validated. The participants in the OCS study possessed similar characteristics to those involved in the qualitative study from which the 
framework was originally developed. The majority of participants in both studies were males, in their 40s, taking combination antiretroviral therapy, who were not currently working. Similarity among the sample participants confirms the types of individuals with whom we validated the dimensions of disability in the Episodic Disability Framework and strengthens the validity of this Framework for use with adults living with HIV with these characteristics. Generalizability of these findings to the broader HIV population should be carefully considered given the OCS sample may under-represent women (17\% in OCS sample versus $22 \%$ in Canada) and Aboriginals (2\% versus $8 \%$ ) living with HIV in Canada [54].

\section{Implications for Practice, Research and Policy}

The Episodic Disability Framework has potential practical implications for clinicians, adults living with HIV, researchers and policy makers. Clinicians can use this Framework to frame their assessments of disability to include physical and mental health symptoms impairments as well as consider a patient's ability to participate in society. Adults living with HIV may use the Framework to articulate their health-related challenges to care providers. Researchers may use the Episodic Disability Framework to guide the development of a future HIV disability questionnaire. Finally, although we did not empirically assess the episodic nature of disability in this study, policy makers might consider using the Framework to recognize the episodic way adults living with HIV might experience disability, which could lead to more flexible income and labor force policies and programs.

\section{Study Limitations}

Our study has several limitations. Results are limited to the type and quality of measured variables available and the characteristics of participants who completed the OCS questionnaire. Perhaps because participants had to be well enough to complete a 90 to 120 minute interview, 
the spectrum of respondents reporting poor health was limited. Furthermore, all respondents were residents of the Greater Toronto Area, Ontario and the majority was taking antiretroviral medications. Generalization to other individuals and settings remains to be determined. Second, ceiling effects of indicator variables and the absence of uncertainty, a key dimension of disability limited our ability to validate the Episodic Disability Framework. Future research should include careful consideration of the distribution of variables prior to their inclusion in the measurement model and include measures of uncertainty to validate this dimension of the Framework. Third, a cross-sectional analysis such as this is unable to validate the episodic nature of disability. Longitudinally exploring the episodic nature of disability experienced by adults living with HIV is vitally important, given the importance of time in our Framework. Fourth, while our results indicate that dimensions of disability are correlated, the direct relationships between the dimensions are unknown. Our results provide a measurement model which can serve as the foundation for future structural equation model analyses to determine the relationships between the dimensions of disability and whether they differ based on personal or disease characteristics for adults living with HIV. Next, this study specifically focused on validating the first component of the Episodic Disability Framework, dimensions of disability. Further empirical testing is needed to validate the contextual factor and trigger components of the Framework and determine their relationships with dimensions of disability. Finally, our aim was to validate dimensions of disability within the Episodic Disability Framework. We used available data from a rich source of socio-behavioral variables collected in an observational cohort study with adults living with HIV. However, given existing measures in the OCS questionnaire were neither developed, nor validated for the purpose of describing disability, future research will involve the development of a new HIV-specific measure of disability using 
content from the newly validated dimensions of disability in the Framework.

\section{CONCLUSIONS}

Results from this confirmatory factor analysis support the validity of four dimensions of disability experienced by adults living with HIV in the Episodic Disability Framework. This is the first known conceptual framework of disability developed from the perspective of adults living with HIV. The Episodic Disability Framework can be used to help describe the multidimensional nature of disability experienced by adults living with HIV and can lay the foundation for developing a future HIV disability measure for clinical and health services research.

\section{ACKNOWLEDGEMENTS}

We gratefully acknowledge the Ontario HIV Treatment Network and the OCS Study Team. We thank Dr. Ann Burchell for reviewing an earlier draft of this manuscript.

*The OHTN Cohort Study Research Team consists of Dr. Sean B. Rourke (Principal Investigator, University of Toronto and OHTN), Dr. Ann Burchell (Co-Principal Investigator, OHTN), Dr. Sandra Gardner (OHTN), Dr. Sergio Rueda (OHTN), Dr. Ahmed Bayoumi and Dr. Kevin Gough, St. Michael's Hospital; Dr. Jeffrey Cohen, Windsor Regional Hospital; Dr. Curtis Cooper, Ottawa General Hospital; Dr. Don Kilby, University of Ottawa Health Services; Dr. Mona Loutfy and Dr. Fred Crouzat,, Maple Leaf Medical Clinic; Dr. Anita Rachlis and Dr. Nicole Mittmann, Sunnybrook Health Sciences Centre; Dr. Janet Raboud and Dr. Irving Salit, Toronto General Hospital; Dr. Edward Ralph, St. Joseph’s Health Care; Dr. Roger Sandre, 
Sudbury Regional Hospital; Dr. Marek Smieja, Hamilton Health Sciences, McMaster University Medical Centre; and Dr. Wendy Wobeser, Hotel Dieu Hospital.

We gratefully acknowledge all of the people living with HIV who volunteered to participate in the OHTN Cohort Study and the work and support of the past and present members of the OCS Governance Committee: Darien Taylor, Dr. Evan Collins, Dr. Greg Robinson, Shari Margolese, Patrick Cupido, Tony Di Pede, Rick Kennedy, Michael Hamilton, Ken King, Brian Finch, Lori Stoltz, Adrian Betts, Colleen Price, Tracey Conway, John MacTavish, Claire Kendall, Anita Benoit, Rosie Thein, Brian Huskins, Les Bowman, Dr. Ahmed Bayoumi, Dr. Clemon George, and Dr. Curtis Cooper. We thank all the interviewers, data collectors, research associates and coordinators, nurses and physicians who provide support for data collection and extraction. The authors wish to thank the OHTN staff and their teams for data management and IT support (Mark Fisher, Director, IT), and OCS project coordination (Brooke Ellis, Coordinator, OCS Research). We also acknowledge the Public Health Laboratories, Public Health Ontario, for supporting record linkage with the HIV viral load database. The OHTN Cohort Study is supported by the Ontario Ministry of Health and Long-Term Care. The opinions, results and conclusions are those of the authors and no endorsement by the Ontario HIV Treatment Network is intended or should be inferred.

\section{DECLARATION OF INTEREST}

Kelly O'Brien was supported by a Fellowship from the Canadian Institutes of Health Research (CIHR), HIV/AIDS Research Program and Michael G. DeGroote Postdoctoral Fellowship (McMaster University). Dr. Ahmed Bayoumi was supported by a Canadian Institutes of Health Research / Ontario Ministry of Health and Long-Term Care Applied Chair in Health Services 
and Policy Research. The Centre for Research on Inner City Health was supported in part by a grant from the Ontario Ministry of Health and Long-Term Care. The views expressed in this article are those of the authors, and no official endorsement by supporting agencies is intended or should be inferred. Trevor A. Hart is supported by a CIHR New Investigator Salary Award. 


\section{REFERENCES}

1. Murphy, E.L., et al., Highly active antiretroviral therapy decreases mortality and morbidity in patients with advanced HIV disease. Ann Intern Med, 2001. 135(1): p. 1726.

2. Palella, F.J., Jr., et al., Declining morbidity and mortality among patients with advanced human immunodeficiency virus infection. HIV Outpatient Study Investigators. N Engl J Med, 1998. 338(13): p. 853-60.

3. Weiss, J.J., et al., Prevalence and patient awareness of medical comorbidities in an urban AIDS clinic. AIDS Patient Care STDS, 2010. 24(1): p. 39-48.

4. Willard, S., et al., Does "asymptomatic" mean without symptoms for those living with HIV infection? AIDS Care, 2009. 21(3): p. 322-8.

5. Rusch, M., et al., Impairments, activity limitations and participation restrictions: prevalence and associations among persons living with HIV/AIDS in British Columbia. Health Qual Life Outcomes, 2004. 2: p. 46.

6. Stanton, D.L., et al., Functional status of persons with HIV infection in an ambulatory setting. J Acquir Immune Defic Syndr, 1994. 7(10): p. 1050-6.

7. Gaidhane, A.M., et al., Assessing self-care component of activities and participation domain of the international classification of functioning, disability and health (ICF) among people living with HIV/AIDS. AIDS Care, 2008: p. 1-7.

8. Meyer-Rosberg, K., et al., Peripheral neuropathic pain--a multidimensional burden for patients. Eur J Pain, 2001. 5(4): p. 379-89.

9. Blanch, J., et al., Impact of lipodystrophy on the quality of life of HIV-1-infected patients. J Acquir Immune Defic Syndr, 2002. 31(4): p. 404-7. 
10. Dray-Spira, R., et al., Employment loss following HIV infection in the era of highly active antiretroviral therapies. Eur J Public Health, 2006. 16(1): p. 89-95.

11. O'Brien, K.K., et al., Exploring disability from the perspective of adults living with HIV/AIDS: development of a conceptual framework. Health Qual Life Outcomes, 2008. 6: p. 76.

12. O'Brien, K.K., et al., Putting episodic disability into context: a qualitative study exploring factors that influence disability experienced by adults living with HIV/AIDS. J Int AIDS Soc, 2009. 12(1): p. 5.

13. Fougeyrollas, P., et al., The handicap creation process: analysis of the consultation. ICIDH International Network, 1991. 4: p. 8-37.

14. Nagi, S., Some conceptual issues in disability and rehabilitation, in Sociology and Rehabilitation, M. Sussman, Editor 1965, American Sociological Association: Washington, DC. p. 100-113.

15. Nagi, S., Disability concepts revisited: implications for prevention, in Disability in America: Toward a National Agenda for Prevention, A. Pope and A. Tarlov, Editors. 1991, National Academy Press: Washington, DC. p. 309-327.

16. Verbrugge, L.M. and A.M. Jette, The disablement process. Soc Sci Med, 1994. 38(1): p. 1-14.

17. World Health Organization, International classification of impairments, disabilities and handicaps (ICIDH)1980, Geneva: The World Health Organization.

18. World Health Organization, International classification of functioning, disability and health (ICF)2001, Geneva.: The World Health Organization. 
19. Rourke, S.B., et al., Cohort Profile: The Ontario HIV Treatment Network Cohort Study (OCS). Int J Epidemiol, 2012.

20. Ontario HIV Treatment Network. OHTN Cohort Study. 2010; Available from: cited 2011 Feb. Available From: http://www.ohtn.on.ca/Pages/Research/OHTN-Cohort-Study.aspx.

21. Ware, J.E., Jr. and B. Gandek, Overview of the SF-36 Health Survey and the International Quality of Life Assessment (IQOLA) Project. J Clin Epidemiol, 1998. 51(11): p. 903-12.

22. Horsman, J., et al., The Health Utilities Index (HUI): concepts, measurement properties and applications. Health Qual Life Outcomes, 2003. 1: p. 54.

23. Radloff, L.S., The CES-D scale: A self-report depression scale for research in the general population. Applied Psychological Measurement, 1977. 1(3): p. 385-401.

24. McHorney, C.A., J.E. Ware, Jr., and A.E. Raczek, The MOS 36-Item Short-Form Health Survey (SF-36): II. Psychometric and clinical tests of validity in measuring physical and mental health constructs. Med Care, 1993. 31(3): p. 247-63.

25. McHorney, C.A., et al., The MOS 36-item Short-Form Health Survey (SF-36): III. Tests of data quality, scaling assumptions, and reliability across diverse patient groups. Med Care, 1994. 32(1): p. 40-66.

26. Justice, A.C., et al., Development and validation of a self-completed HIV symptom index. J Clin Epidemiol, 2001. 54 Suppl 1: p. S77-90.

27. Brooks, R., R. Rabin, and F. Charro, The measurement and valuation of health status using EQ-5D: A European perspective.2003, Dordrecht, The Netherlands: Kluwer Academic Publishers. 
28. Statistics Canada, National population health survey household component documentation for the derived variables and the constant longitudinal variables (specifications): Cycles 1 to 7 (1994/1995 to 2006/2007). 2008, Ottawa: Statistics Canada.

29. Wu, A.W., et al., Evidence for reliability, validity and usefulness of the Medical Outcomes Study HIV Health Survey (MOS-HIV). Qual Life Res, 1997. 6(6): p. 481-93.

30. Lyon, D.E. and J.B. Younger, Purpose in life and depressive symptoms in persons living with HIV disease. J Nurs Scholarsh, 2001. 33(2): p. 129-33.

31. Burack, J.H., et al., Depressive symptoms and CD4 lymphocyte decline among HIVinfected men. JAMA, 1993. 270(21): p. 2568-73.

32. Chishinga, N., et al., Validation of brief screening tools for depressive and alcohol use disorders among TB and HIV patients in primary care in Zambia. BMC Psychiatry, 2011. 11: p. 75 .

33. Lyketsos, C.G., et al., Depressive symptoms as predictors of medical outcomes in HIV infection. Multicenter AIDS Cohort Study. JAMA, 1993. 270(21): p. 2563-7.

34. Cook, J.A., et al., Depressive symptoms and AIDS-related mortality among a multisite cohort of HIV-positive women. Am J Public Health, 2004. 94(7): p. 1133-40.

35. Furlong, W.J., et al., The Health Utilities Index (HUI) system for assessing health-related quality of life in clinical studies. Ann Med, 2001. 33(5): p. 375-84.

36. Ware, J.E., Jr., SF-36 health survey update. Spine (Phila Pa 1976), 2000. 25(24): p. 31309.

37. Canadian Institute for Health Information, Definitions and data sources. Health Indicators, 2003. 2: p. 4-41. 
38. Revicki, D.A., K. Chan, and F. Gevirtz, Discriminant validity of the Medical Outcomes Study cognitive function scale in HIV disease patients. Qual Life Res, 1998. 7(6): p. 5519.

39. Mathews, W.C. and S. May, EuroQol (EQ-5D) measure of quality of life predicts mortality, emergency department utilization, and hospital discharge rates in HIV-infected adults under care. Health Qual Life Outcomes, 2007. 5: p. 5.

40. $\mathrm{Wu}, \mathrm{A} . \mathrm{W}$., et al., Validity and responsiveness of the euroqol as a measure of healthrelated quality of life in people enrolled in an AIDS clinical trial. Qual Life Res, 2002. 11(3): p. 273-82.

41. O'Brien, K.K., et al., How do existing HIV-specific instruments measure up? Evaluating the ability of instruments to describe disability experienced by adults living with HIV. Health Qual Life Outcomes, 2010. 8: p. 88.

42. Carter, S.L., et al., Cognitive complaints, depression, medical symptoms, and their association with neuropsychological functioning in HIV infection: a structural equation model analysis. Neuropsychology, 2003. 17(3): p. 410-9.

43. Brown, T.A., Confirmatory factor analysis for applied research2006, New York, NY: The Guilford Press.

44. Kline, R.B., Principles and practice of structural equation modeling2005, New York, NY: The Guilford Press.

45. Muthen, L.K. and B.O. Muthen, Mplus User's Guide 6th Edition1998-2010, Los Angeles, CA.

46. Hu, L.T. and P.M. Bentler, Fit indices in covariance structure modeling: sensitivity to underparameterized model misspecification. Psychol Methods, 1998. 3: p. 424-53. 
47. Hu, L.T. and P.M. Bentler, Cutoff criteria for fit indexes in covariance structure analysis: conventional criteria versus new alternatives. Structural Equation Modeling, 1999. 6(1$55)$.

48. Rigdon, E.E., CFI versus RMSEA: A comparison of two fit indexes for structural equation modeling. Structural Equation Modeling, 1996. 3(4): p. 369-79.

49. Joreskog, K.G., Testing structural equation models, in Testing structural equation models, K.A. Bollen and J.S. Long, Editors. 1993, Sage: Newbury Park, CA. p. 294-316.

50. Wu, A.W., et al., A health status questionnaire using 30 items from the Medical Outcomes Study. Preliminary validation in persons with early HIV infection. Med Care, 1991. 29(8): p. 786-98.

51. Vidrine, D.J., et al., Assessing a conceptual framework of health-related quality of life in a HIV/AIDS population. Qual Life Res, 2005. 14(4): p. 923-33.

52. Sousa, K.H. and O.M. Kwok, Putting Wilson and Cleary to the test: analysis of a HRQOL conceptual model using structural equation modeling. Qual Life Res, 2006. 15(4): p. 725-37.

53. Rourke, S.B., M.H. Halman, and C. Bassel, Neurocognitive complaints in HIV-infection and their relationship to depressive symptoms and neuropsychological functioning. J Clin Exp Neuropsychol, 1999. 21(6): p. 737-56.

54. Public Health Agency of Canada, HIV Epi Updates. July 2010, 2010, Surveillance and Risk Assessment Division, Centre for Communicable Diseases and Infection Control, Public Health Agency of Canada. 
Table 1: Characteristics of Participants $(n=913)$

\begin{tabular}{|c|c|}
\hline Characteristic & Number (\%) \\
\hline \multicolumn{2}{|l|}{ Gender } \\
\hline Male & $757(83 \%)$ \\
\hline Female & $153(17 \%)$ \\
\hline Other* & $3(<1 \%)$ \\
\hline Age (median; IQR) & 47 years $(41-53$ years $)$ \\
\hline Length of Time Since Diagnosis (median; IQR) & 11 years $(6-17$ years $)$ \\
\hline Taking Antiretrovirals & $783(86 \%)$ \\
\hline \multicolumn{2}{|l|}{ Race } \\
\hline White & $588(64 \%)$ \\
\hline Black / African & $158(17 \%)$ \\
\hline Asian / Latin American / Arab & $101(11 \%)$ \\
\hline Aboriginal & $22(2 \%)$ \\
\hline \multicolumn{2}{|l|}{ Employment Status } \\
\hline Employed (full time or part time) & $412(45 \%)$ \\
\hline On disability leave from work & $289(32 \%)$ \\
\hline Student or retired & $106(12 \%)$ \\
\hline Unemployed & $79(9 \%)$ \\
\hline Volunteering & $25(3 \%)$ \\
\hline \multicolumn{2}{|l|}{ Educational Level } \\
\hline No formal education or less than grade 9 & $32(3 \%)$ \\
\hline Partially or fully completed high school education & $268(29 \%)$ \\
\hline Partially or fully completed trade or technical training & $31 \quad(3 \%)$ \\
\hline Partially or fully completed college & $234(26 \%)$ \\
\hline Partial or fully completed university & $279(31 \%)$ \\
\hline Post-graduate education & $69 \quad(8 \%)$ \\
\hline \multicolumn{2}{|l|}{ Gross Personal Income (Canadian dollars) } \\
\hline Less than $\$ 20,000$ per year & $386(43 \%)$ \\
\hline$\$ 20,000$ to less than $\$ 40,000$ per year & $214(24 \%)$ \\
\hline$\$ 40,000$ to less than $\$ 60,000$ per year & $140(16 \%)$ \\
\hline$\$ 60,000$ to less than $\$ 80,000$ per year & $70 \quad(8 \%)$ \\
\hline$\$ 80,000$ or more per year & $85(9 \%)$ \\
\hline
\end{tabular}


$\mathrm{IQR}=$ interquartile range. Not all characteristics will add to the total $\mathrm{n}$ due to missing responses.

*Other=trans, inter-sexed or other. 


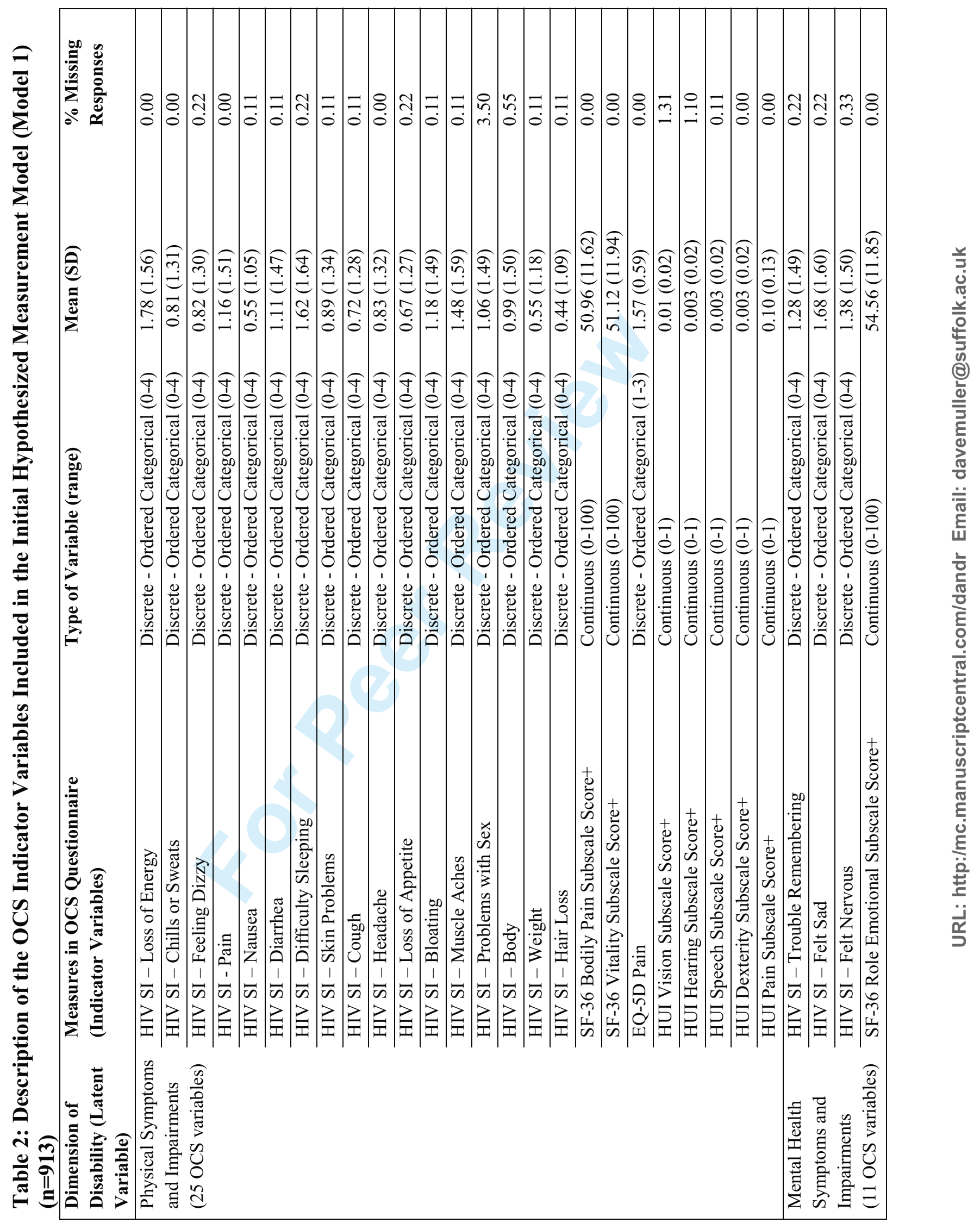




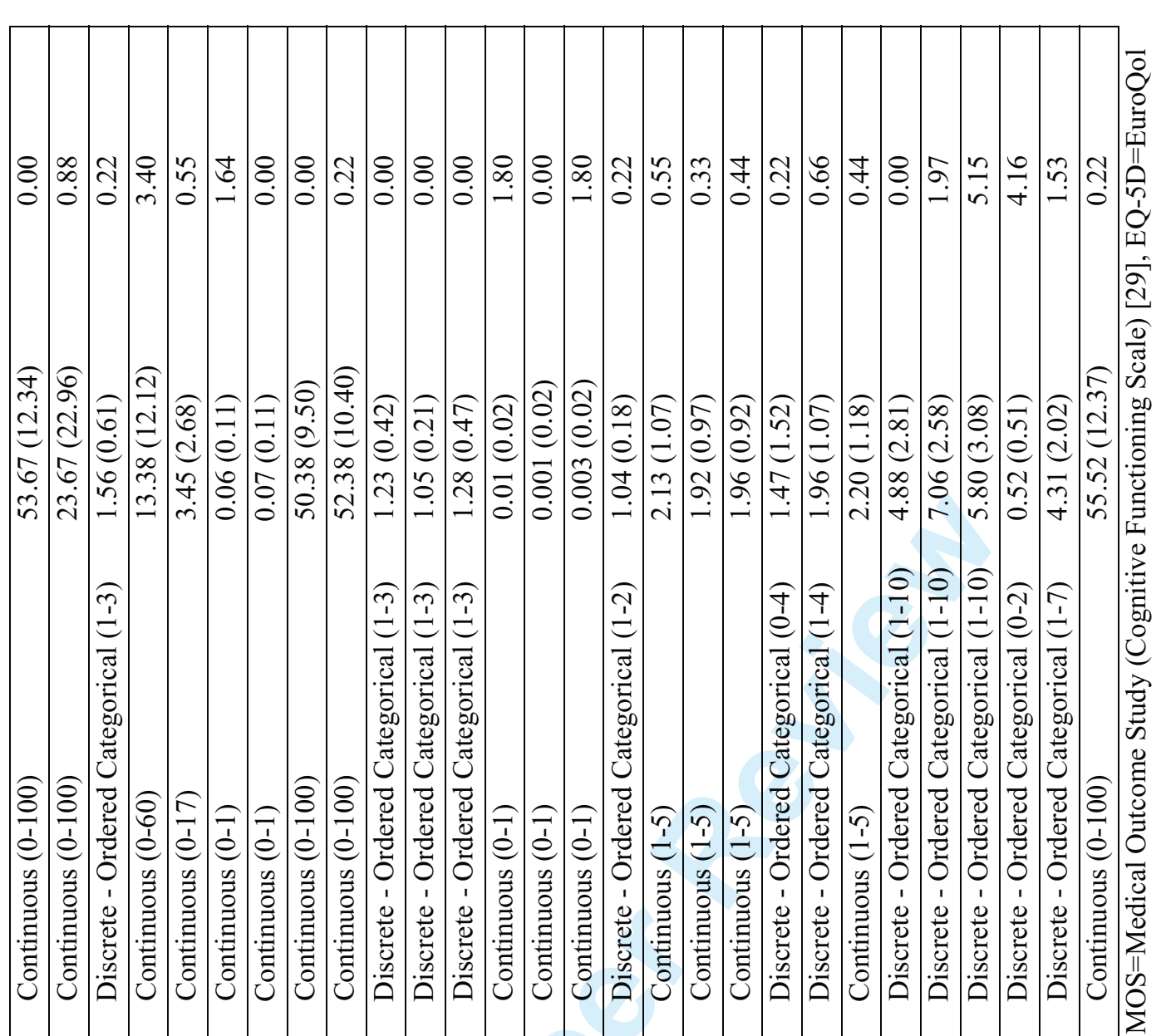

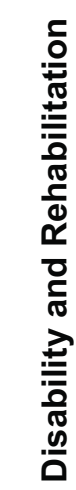

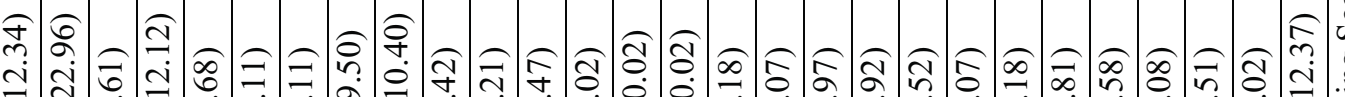

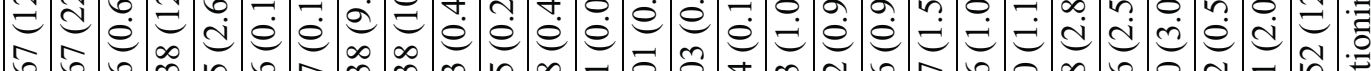

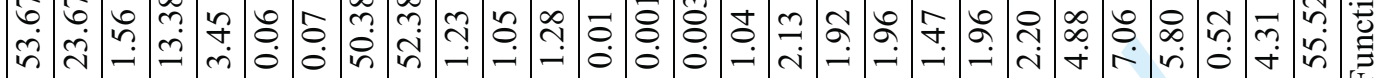

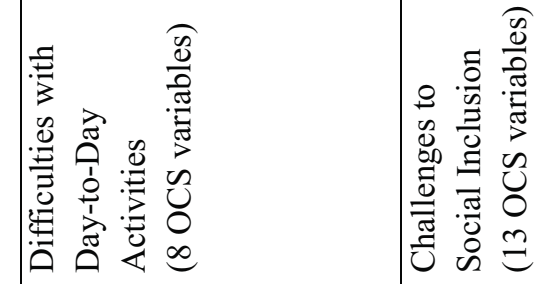




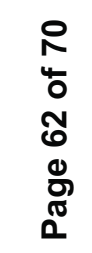

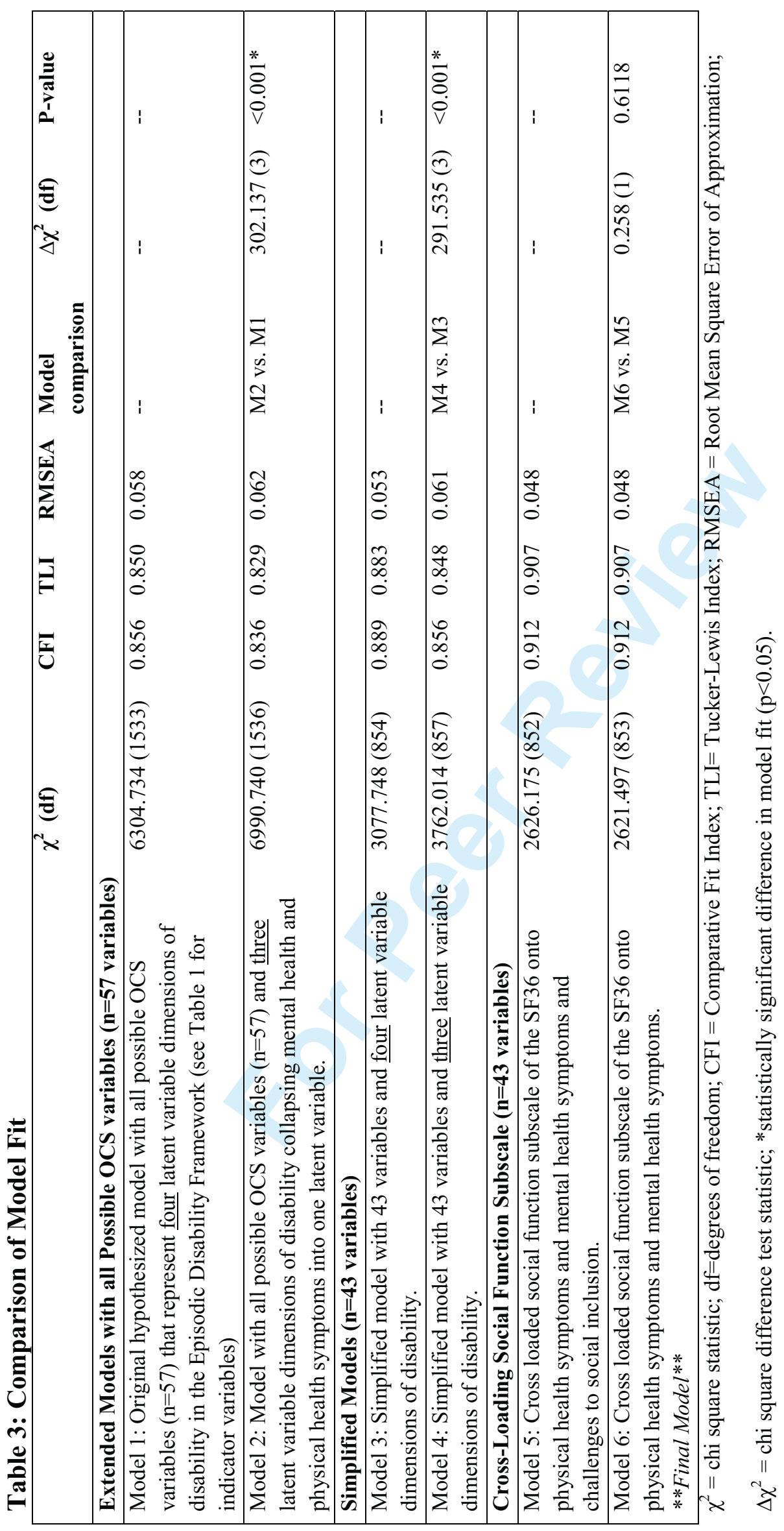

- Nmナம 
足

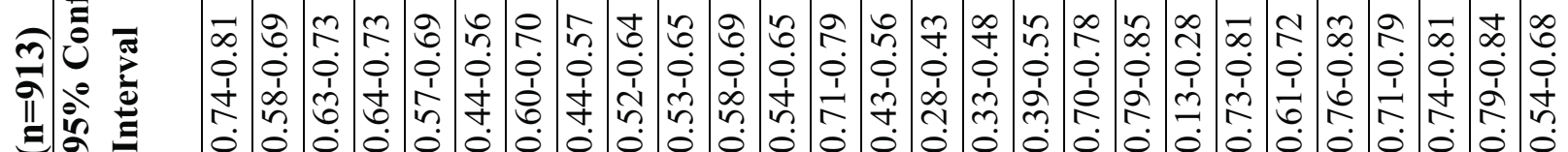

ㄱ.

$\mid$\begin{tabular}{l|l|l} 
& &
\end{tabular}

(1)

$\mid$

(

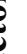

ฆ

远

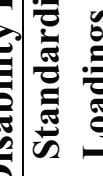

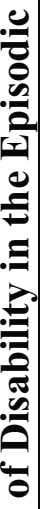

(a)

(⿳)十亏

|

$11 . \equiv$

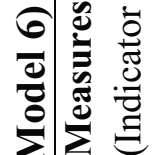

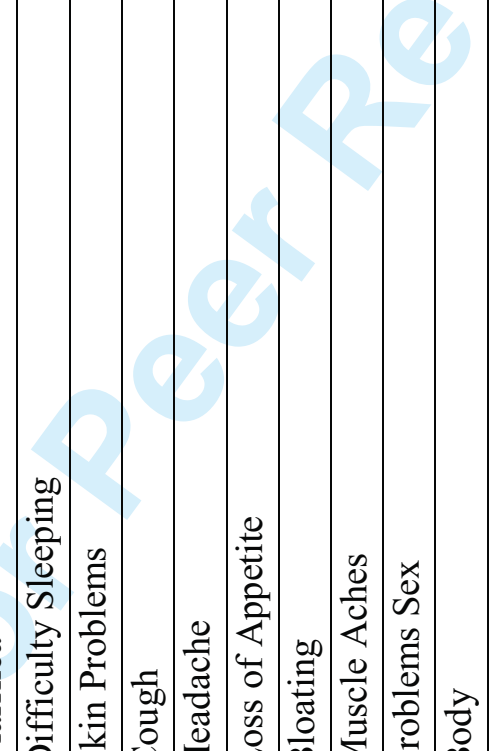

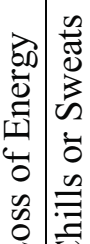

:

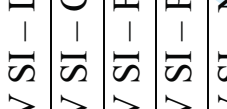

0

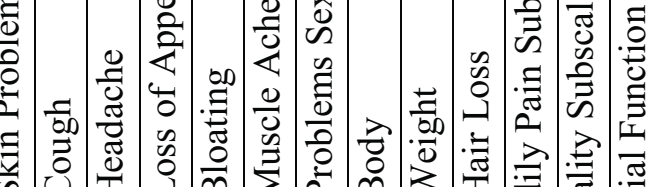

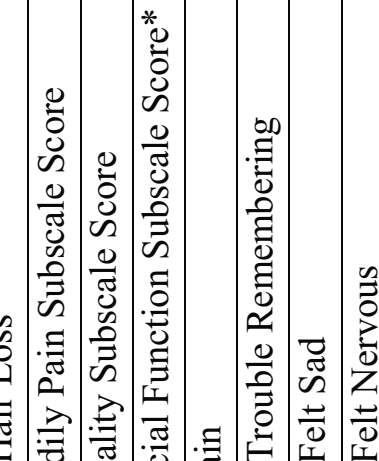

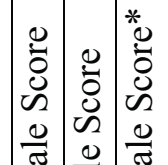

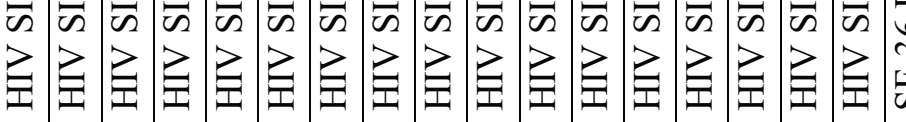

先
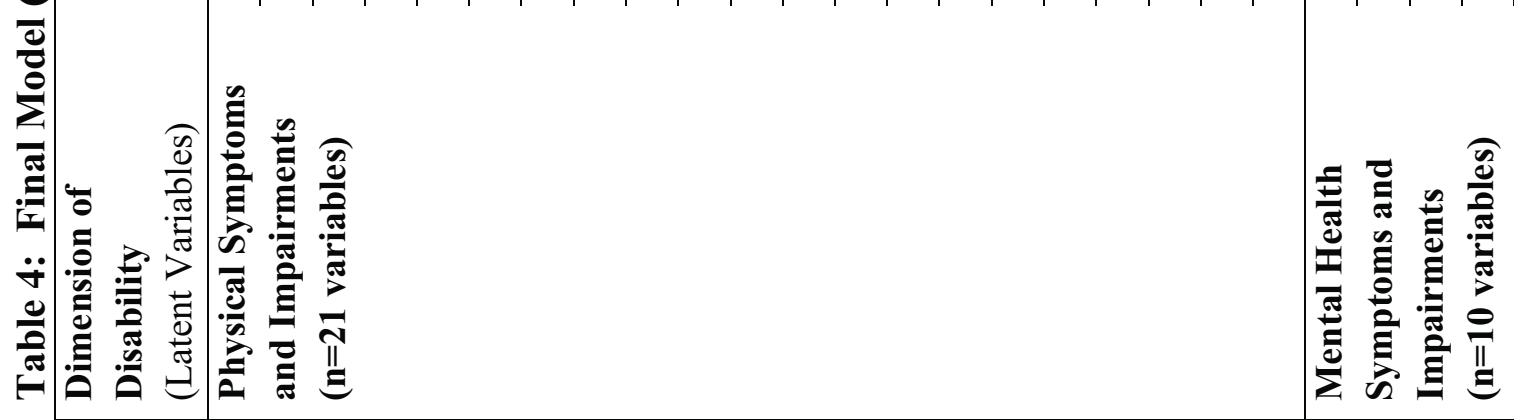
8
4
0
8
0
0
8
0
0

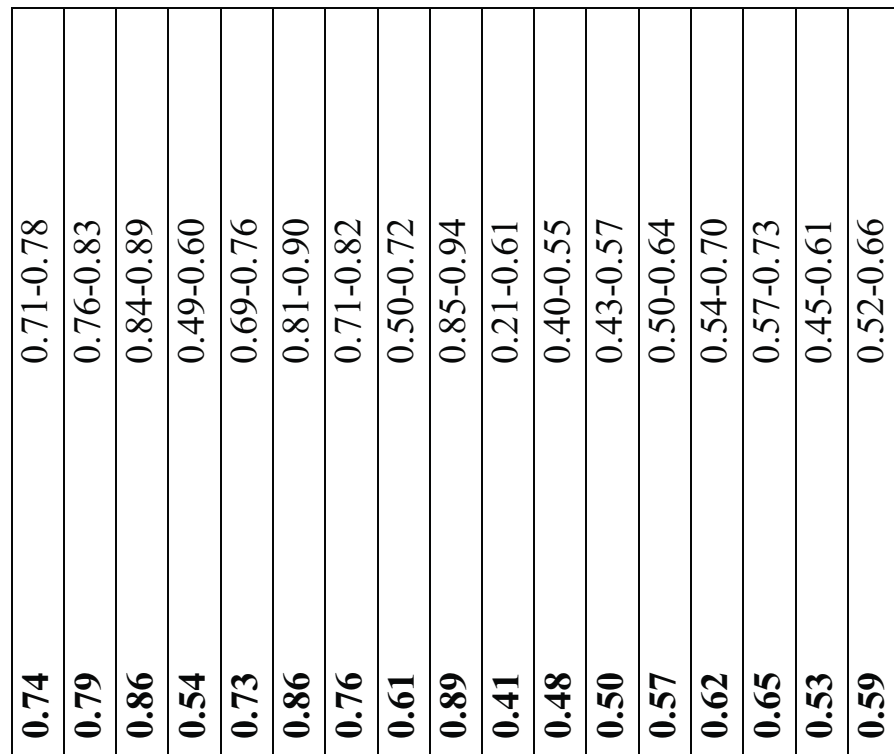

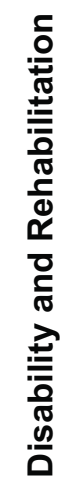

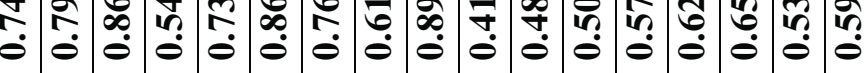
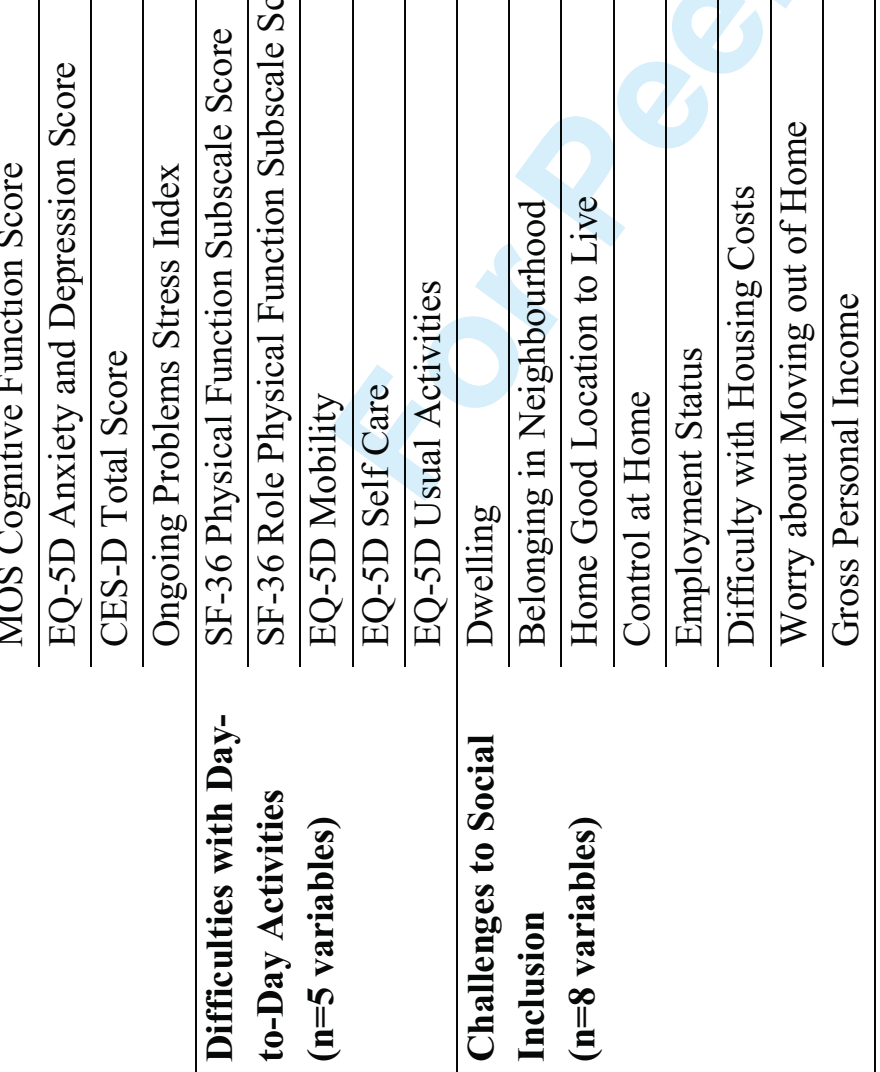

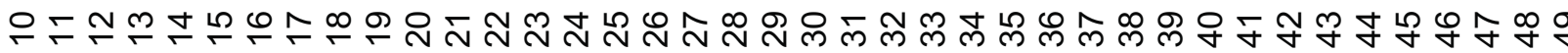




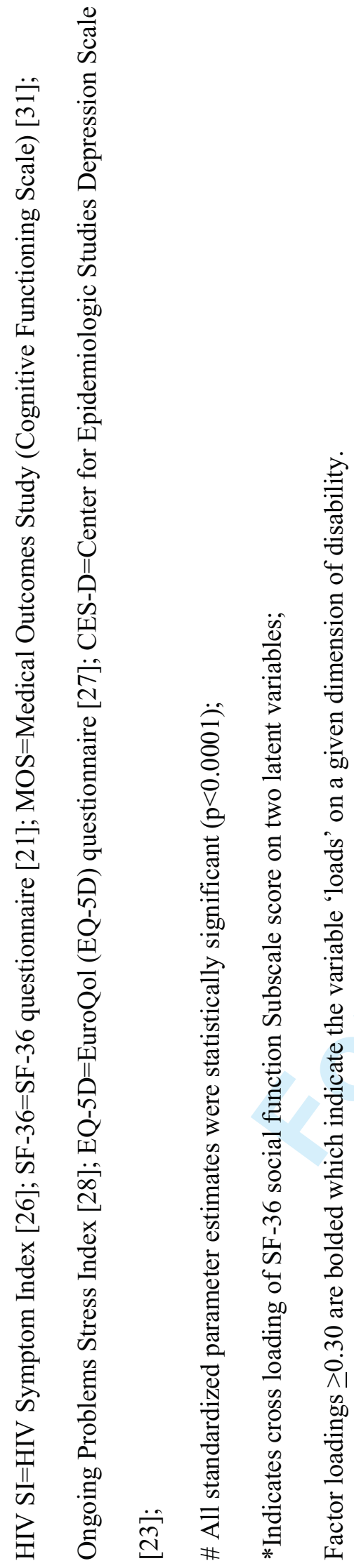


$R$
$\stackrel{2}{0}$
0
8
0
8
8
0
0

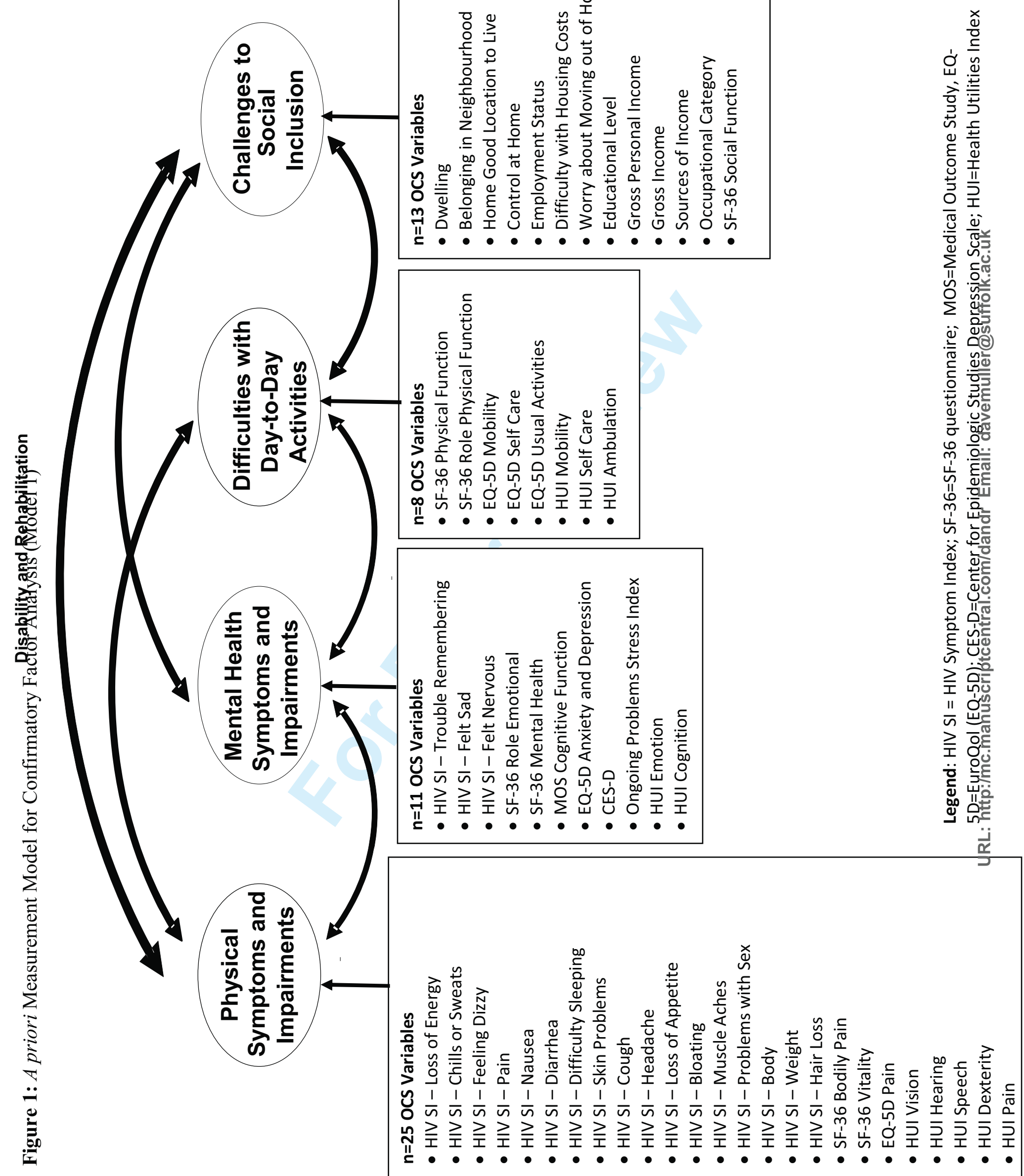

- Nm+L 


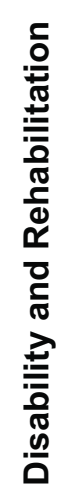

음

先

흠

है.

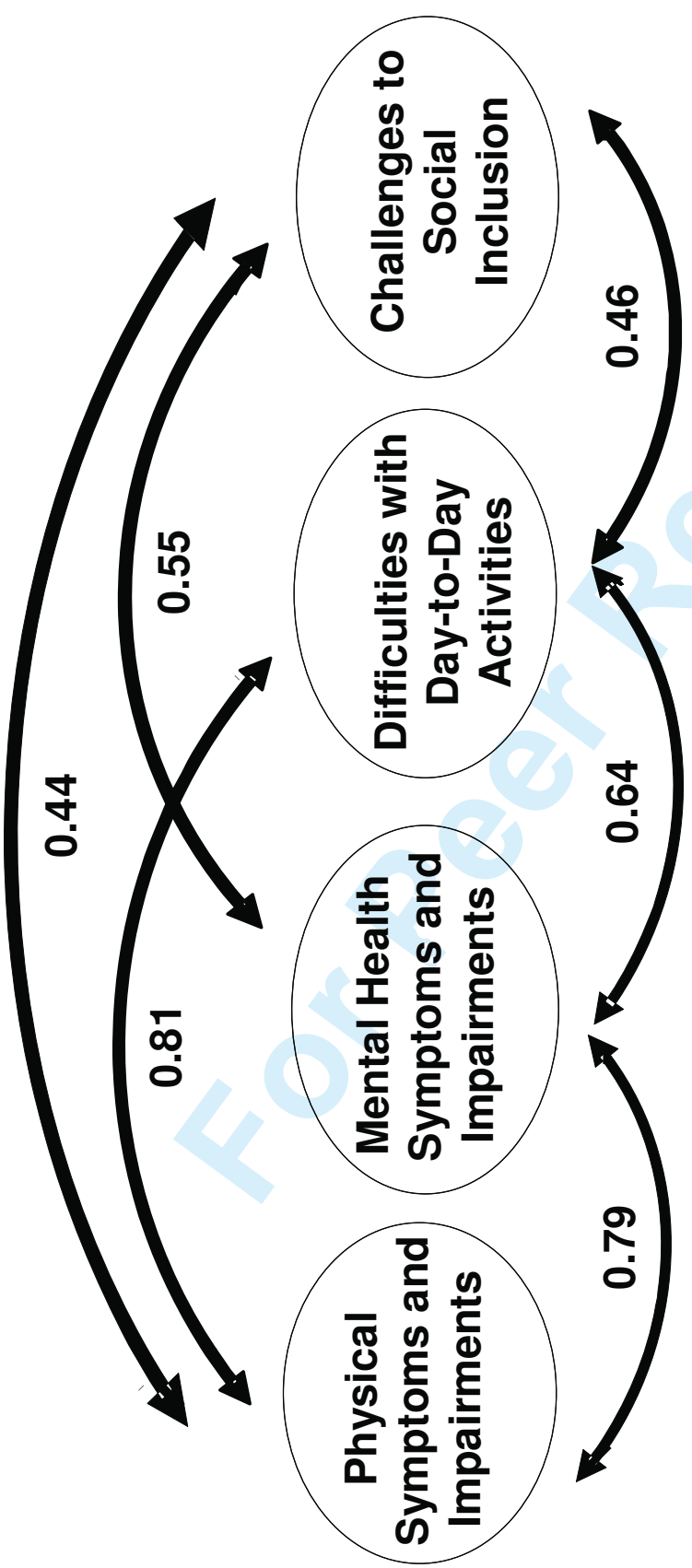

$R$
4
0
0
0
0
0
0
0

드

晃

- Nmナம 